\title{
Modeling and Simulation of Fast Fading Channels in Indoor Peer-to-Peer Scenarios
}

\author{
Evgenii Vinogradov ${ }^{1}$, Wout Joseph ${ }^{2}$, Claude Oestges ${ }^{1}$ \\ ${ }^{1}$ ICTEAM - Electrical Engineering, Universite catholique de Louvain, Louvain-la-Neuve, Belgium \\ ${ }^{2}$ Dept. of Information Technology, Ghent University/IMinds INTEC-WICA, Ghent, Belgium
}

\section{INTRODUCTION}

Traditional cellular solutions are coming under increasing pressure from wireless peer-to-peer (P2P) systems. The growing interest in wireless indoor communication systems has resulted in many investigations on the characteristics of indoor radio propagation channels. In [1], various properties of indoor peer-to-peer channels have been analyzed for static nodes only. In [2], peer-to-peer channels were investigated in a typical US office environment, consisting in a large indoor area containing individual cubicle-style offices. The same environment was also used in [3] to investigate the multi-user separation in duallink MIMO indoor scenarios.

In this paper, we investigate narrowband indoor distributed channels based on an experimental campaign in an office environment. We evaluate the fast fading statistics using the second-order scattering fading (SOSF) distribution [4], [2]. It turns out from the measurements that the fading statistics change over time. An approach to model these effects is proposed.

\section{Measurements}

Our results are based on indoor peer-to-peer measurements in an office environment consisting in different rooms aligned along a corridor and separated by different types of walls. Mobile nodes were either randomly moved over a small scale (within a square of $1 \mathrm{~m}^{2}$ ), or over a larger scale (throughout the whole room they were in). The measurements were carried out with UCL/ULB Elektrobit CS channel sounder at a carrier frequency of $3.8 \mathrm{GHz}$ [5].

Following the concept of [2], the channel can be seen as a superposition of path loss, static shadowing, dynamic shadowing, and fast fading. The path loss and shadowing were removed to obtain only fast fading component of the channels.

Our goal is to create a model which generates fast fading realizations taking into account both first- and second-order statistics. However, measurement parameters do not permit us to extract appropriate second-order statistics from samples. For this reason, we need to derive second order statistics analytically,using an equivalent reference model.

\section{TIME-VARIANT FAST FADING STATISTICS}

It is shown in [2] that for peer-to-peer environments fastfading amplitude can be described by a single distribution including a weighted combination a line-of-sight (LOS) component, a Rayleigh fading component and a Double Rayleigh

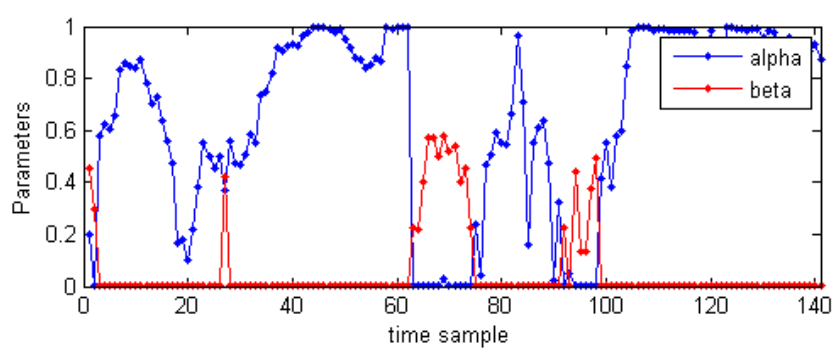

Fig. 1. Example of fast-fading distribution parameters over time for double mobile small-scale motion.

fading component. Hence, any realization of the channel can be expressed as

$$
g=\omega_{0} e^{j \theta}+\omega_{1} G_{1}+\omega_{2} G_{2} G_{3}
$$

where $G_{1}, G_{2}, G_{3}$ are i.i.d. complex normal random variables with zero mean and unit variance, and $\theta$ is a constant phase shift angle in $[0,2 \pi]$. The probability density function of $r=|g|$ is given as shown in [6] and [2] by the socalled second order scattering fading (SOSF) distribution. The distribution can be specified by two parameters $\alpha=\frac{\omega_{2}^{2}}{\omega_{0}^{2}+\omega_{1}^{2}+\omega_{2}^{2}}$ and $\beta=\frac{\omega_{0}^{2}}{\omega_{0}^{2}+\omega_{1}^{2}+\omega_{2}^{2}}$ [7]. Values of the parameters can be partitioned into five groups: (i) Rician $(\alpha=0, \beta>0$, with $K$ Factor $\left.K=\frac{\beta}{1-\beta}\right)$, (ii) Rayleigh $(\alpha=0, \beta=0)$, (iii) Double Rayleigh (DR) $(\alpha=1, \beta=0)$, (iv) Double Rayleigh under LOS conditions (DRLOS) $(\alpha+\beta=1, \alpha<1, \beta<1)$, (v) Rayleigh-Double-Rayleigh (RDR) subset $(0<\alpha<1, \beta=0)$.

It turns out from measurements that the parameters $\alpha$ and $\beta$ for peer-to-peer channels depend on time. Looking at the variability of the parameters for an exemplary double-mobile channel (with small-scale motion) in Fig. 1, we see that predominantly Rayleigh-Double-Rayleigh fading occurs. Only scarcely, we observe other kinds of fading. For single-mobile channels, we observe Rayleigh fading and Rician fading. It turns out that the distribution of the parameter $\alpha$ for the RDR subset can be well approximated by the Beta distribution. For the DRLOS subset, we define $\Delta=\sqrt{(1-\alpha)^{2}+\beta^{2}}$. It turns out that $\Delta$ is Beta-distributed. Finally, the distribution of the K-Factor $K=\frac{\beta}{1-\beta}$ can be modeled by Extremal Value Distribution. By that, we can estimate a probability distribution of the SOSF parameters within the subset. A summary of these parameters evaluated from the measurements is provided in Table I. 
TABLE I

EVAluAted PARAMETERS OF THE SOSF Distribution FOR DiFFERENT MOBILITY

\begin{tabular}{l|c|c}
\hline \multirow{2}{*}{ Subset } & Small scale motion & Large scale motion \\
\cline { 2 - 3 } & Distribution & Distribution \\
\hline \multicolumn{2}{|c}{ Single mobile } \\
\hline Rician & $K \sim p_{\text {ev }}(-0.8,3), \alpha=0$ & $K \sim p_{\text {ev }}(-0.44,2.8), \alpha=0$ \\
\hline \hline & \multicolumn{2}{c}{ Double mobile } \\
\hline Rician & $K \sim p_{\text {ev }}(-1.4,3.5), \alpha=0$ & $K \sim p_{\text {ev }}(-2.4,2.6), \alpha=0$ \\
RDR & $\alpha \sim p_{\beta}(2,0.85), \beta=0$ & $\alpha \sim p_{\beta}(2.4,0.7), \beta=0$ \\
DRLOS & $\Delta \sim p_{\beta}(1,0.85)$ & $\Delta \sim p_{\beta}(1.9,3.5)$ \\
\hline
\end{tabular}

TABLE II

TRANSITION MATRICES FOR SINGLE MOBILE SUBSCENARIOS

\begin{tabular}{l|cc|cc}
\hline & \multicolumn{2}{|c}{ Probability } & \multicolumn{2}{c}{ Transition matrix } \\
\hline Subset & Small Scale & Large Scale & Rician & Rayleigh \\
\hline Rician & 0.25 & 0.16 & 0.77 & 0.23 \\
Rayleigh & 0.75 & 0.84 & 0.06 & 0.94 \\
\hline
\end{tabular}

Modeling transitions between different fading distributions can be solved by using a hidden Markov model (HMM) [8]. As an example, the state transition probabilities for single mobile scenarios, estimated from the measurements, are presented in Table II

Using the Double Ring geometry (Fig. 2) described in [9], we are able to derive a reference model for SOSF. Next, using the model, the autocorrelation function of SOSF can be expressed as

$$
\begin{aligned}
R_{g g}(\tau)= & \mathbb{E}\left[g(t) g^{*}(t+\tau)\right]= \\
& \left(\omega_{1}^{2}+\omega_{2}^{2}\right) J_{0}\left(2 \pi f_{T} \tau\right) J_{0}\left(2 \pi f_{R} \tau\right) \\
& +\omega_{0}^{2} \cos \left(2 \pi f_{r e l} \cos \left(\Theta_{r e l}\right)\right)
\end{aligned}
$$

where $f_{T}, f_{R}, f_{r e l}$ and $\Theta_{\text {rel }}$ are the maximum Doppler frequencies caused by the movement of the transmitter/the receiver, the relative movement of the nodes and the angle between the relative movement and the LOS component, respectively. By that, we can simulate fast fading reproducing both first- and second-order statistics.

\section{MODEL SUMMARY}

Summarizing, we propose to model small-scale fading with time-variant statistics using following procedure: We start with a random initial state of the HMM. Depending on the state, we set constant values of the SOSF parameters $(\alpha, \beta)$ or check if the state is changed in comparison with the previous one (skipped for the initial state). If the state is unchanged we use an autoregressive model to generate new values of $\alpha, \beta$ or $\Delta$. Otherwise, we draw new values of the parameters using distributions described in Table I. Using $(\alpha, \beta)$, we generate a pre-defined number of complex fading realizations $g(t)$ using the first- and second-order statistics previously derived. Next, the state of the HMM is updated.

\section{CONCLUSIONS}

This paper has presented an analysis and an empirical model of time-variant fading statistics of a peer-to-peer network

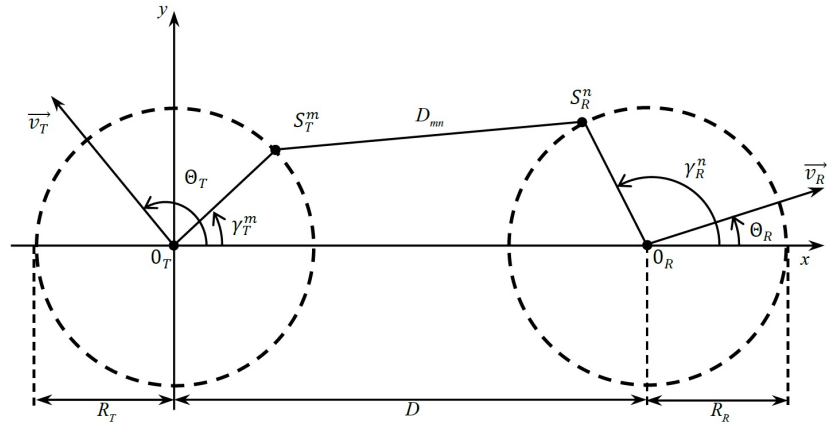

Fig. 2. The geometrical double-ring model with local scatterers around a mobile transmitter(left) and a mobile receiver(right)

based on measurement in an indoor office environment at 3.8 GHz: (i) Models and simulators for second-order scattering fading (SOSF) in different mobility scenarios have been proposed; (ii) In double mobile scenarios, the measured data is characterized by the SOSF distribution; (iii) In single mobile scenarios, fast-fading is Rayleigh or Rician distributed; (iv) Distributions of the parameter $\alpha$ for the RDR and $\Delta$ for the DRLOS subsets can be well approximated by the Beta distribution; (v) Distribution of the K-Factor can be modeled by the Extremal Value Distribution; (vi) Transitions between the groups are described by a hidden Markov model.

\section{ACKNOWLEDGEMENTS}

This research has been funded by the Interuniversity Attraction Poles Programme 7/23 BESTCOM initiated by the Belgian Science Policy Office.

\section{REFERENCES}

[1] J. Karedal, A. Johansson, F. Tufvesson, and A. Molisch, "A measurementbased fading model for wireless personal area networks," IEEE Trans. on Wireless Com., vol. 7, no. 11, pp. 4575 - 4585, November 2008.

[2] C. Oestges, N. Czink, B. Bandemer, P. Castiglione, F. Kaltenberger, and A. Paulraj, "Experimental characterization and modeling of outdoor-toindoor and indoor-to-indoor distributed channels," IEEE Transactions on Vehicular Technology, vol. 59, no. 5, pp. 2253 - 2265, June 2010.

[3] N. Czink, B. Bandemer, G. Vazquez-Vilar, L. Jalloul, C. Oestges, and A. Paulraj, "Spatial separation of multi-user MIMO channels," Proc. IEEE Intl. Symposium on PIMRC, pp. 1059 - 1063.

[4] I. Z. Kovacs, P. C. F. Eggers, K. Olesen, and L. G. Petersen, "Investigations of outdoor-to-indoor mobile-to-mobile radio communications channels," Proc. VTC 2002 Fall -IEEE Vehicular Technolgy Conference, pp. $430-434$, September 2002.

[5] F. Sanchez, A. Stephanides, N. Czink, , and C. Oestge, "Fast fading characterization for indoor-to-indoor and outdoor-to-indoor channels," Proc. VTC 2011 FAll - IEEE VT Conf., pp. 1-5, September 2011.

[6] J. Salo, H. M. El-Sallabi, and P. Vainikainen, "Statistical analysis of the multiple scattering radio channel," IEEE Trans. Antennas Propag., vol. 54 no. 11 , pp. 3114 - 3124, November 2006

[7] B. Bandemer, C. Oestges, N. Czink, and A. Paulraj, "Phisically motivated fast-fading model for indoor peer-to-peer channels," Electronic Letters, vol. 45, no. 10 , pp. 515 - 517, May 2009.

[8] Y. Ephraim and N. Merhav, "Hidden markov processes," IEEE Transactions on Inf. Theory, vol. 48, no. 6, pp. 1518 - 1569, June 2002.

[9] C. Patel, G. S. ber, and T. Pratt, "Simulation of rayleigh-faded mobileto-mobile communication channels," IEEE Transactions on Communications, vol. 53, no. 11, pp. 1876 - 1884, November 2005. 